\title{
Public Goods, Social Pressure, and the Choice Between Privacy and Publicity
}

\author{
By Andrew F. Daughety and Jennifer F. Reinganum
}

\section{TECHNICAL APPENDIX}

This technical appendix includes proofs of comparative statics results; the proof of the claim made in the text that if $\beta^{\prime}>\beta$, then $H^{\delta}\left(\delta ; \beta^{\prime}\right)$ first-order stochastic dominates $H^{\delta}(\delta ; \beta)$; computational results, and detailed analysis of the interim preferences over policies.

\section{A. Comparative Statics}

The functions $g^{P}\left(\theta_{i}\right)$ and $g^{O}\left(\theta_{i}\right)$ depend on $\theta_{i}, \beta, \gamma$, and $p$; they are independent of $\alpha$.

Comparative statics of $g^{P}\left(\theta_{i}\right)$. Since $g^{P}\left(\theta_{i}\right)=g_{\text {min }}+\theta_{i}$ and $g_{\text {min }}=\gamma-p$, it is obvious that $g^{P}\left(\theta_{i}\right)$ is an increasing function of $\theta_{i}$, and that the function $g^{P}\left(\theta_{i}\right)$ shifts upward with an increase in $\gamma$, and shifts downward with an increase in $p$. Finally, the function $g^{P}\left(\theta_{i}\right)$ is always independent of $\beta$. Since the utility function is quasilinear, $g^{P}\left(\theta_{i}\right)$ is independent of income, $I$.

Comparative statics of $g^{O}\left(\theta_{i}\right)$. Since $g^{O}(0)=g^{P}(0), g^{O}(0)$ behaves as described above with respect to the parameters. Thus, in what follows, we will consider only $\theta_{i}>0$. Let $R H S \equiv g_{\text {min }}+\theta_{i}+\beta(1-$ $\left.\exp \left[-\left(g^{O}\left(\theta_{i}\right)-g_{\min }\right) / \beta\right]\right)$. For any parameter $m$, the implicit function in Proposition 1(i) can be differentiated to obtain $\partial g^{O} / \partial m=(\partial R H S / \partial m)+\left(\partial R H S / \partial g^{O}\right)\left(\partial g^{O} / \partial m\right)$. Collecting terms implies that $\partial g^{O} / \partial m=(\partial R H S / \partial m) /\left(1-\exp \left[-\left(g^{O}\left(\theta_{i}\right)-g_{\text {min }}\right) / \beta\right]\right)$. Since the denominator is positive, the sign of $\partial g^{O} / \partial m$ is the same as the sign of $(\partial R H S / \partial m)$. To save on notation, it will be useful to define the function $z^{O}\left(\theta_{i}\right) \equiv\left(g^{O}\left(\theta_{i}\right)-g_{\text {min }}\right) / \beta$, and to use $z$ to denote an arbitrary (positive) value.

Since $\partial R H S / \partial \theta_{i}=1$, it follows that $g^{O^{\prime}}\left(\theta_{i}\right)=1 /\left(1-\exp \left[-z^{O}\left(\theta_{i}\right)\right]\right)>0$; that is, the equilibrium action under a policy of publicity (openness) is increasing in type.

Since the parameters $\gamma$ and $p$ appear only in $g_{\text {min }}$, and $\left(\partial R H S / \partial g_{\min }\right)=\left(1-\exp \left[-z^{O}\left(\theta_{i}\right)\right]\right)$, it is straightforward to show that $\partial g^{O}\left(\theta_{i}\right) / \partial g_{\text {min }}=1$. Therefore $\partial g^{O}\left(\theta_{i}\right) / \partial y=1$ and $\partial g^{O}\left(\theta_{i}\right) / \partial p=-1$. 
Differentiating and collecting terms yields $\partial g^{O}\left(\theta_{i}\right) / \partial \beta=\left(1-\exp \left[-z^{O}\left(\theta_{i}\right)\right]-\right.$ $\left.z^{O}\left(\theta_{i}\right) \exp \left[-z^{O}\left(\theta_{i}\right)\right]\right) /\left(1-\exp \left[-z^{O}\left(\theta_{i}\right)\right]\right)$. The function $1-\exp [-z]-z \exp [-z]$ is easily shown to be positive for $z>0$; thus, $\partial g^{O}\left(\theta_{i}\right) / \partial \beta>0$.

Comparative statics of the action differential $\mathrm{g}^{\mathrm{O}}\left(\theta_{\mathrm{i}}\right)-\mathrm{g}^{\mathrm{P}}\left(\theta_{\mathrm{i}}\right)$.

Let $\delta\left(\theta_{i} ; \beta\right) \equiv g^{O}\left(\theta_{i}\right)-g^{P}\left(\theta_{i}\right)=\beta\left(1-\exp \left[-\left(z^{O}\left(\theta_{i}\right)\right]\right)\right.$ denote the action differential as a function of $\theta_{i}$. This difference is increasing in type; that is, $\delta^{\prime}\left(\theta_{i} ; \beta\right)=\exp \left[-\left(z^{O}\left(\theta_{i}\right)\right] g^{O^{\prime}}\left(\theta_{i}\right)>0\right.$. Thus, the highest type inflates his action the most. We have already seen that $\partial g^{O}\left(\theta_{i}\right) / \partial g_{\text {min }}=1$; this yields the immediate result that $\partial z^{O}\left(\theta_{i}\right) / \partial g_{\text {min }}=\left(\partial\left(g^{O}\left(\theta_{i}\right)-g_{\text {min }}\right) / \partial g_{\text {min }}\right) / \beta=0$. This implies that the action differential $\delta\left(\theta_{i} ; \beta\right)$ is independent of the parameters $\gamma$ and $p$. Since $g^{P}\left(\theta_{i}\right)$ is independent of $\beta$, then $\partial \delta\left(\theta_{i} ; \beta\right) / \partial \beta=\partial g^{O}\left(\theta_{i}\right) / \partial \beta>0$

B. Proof of Claim that if $\beta^{\prime}>\beta$, then $H^{\delta}(\delta ; \beta)$ First-order Stochastic Dominates $H^{\delta}(\delta ; \beta)$

Recall that $\delta(\theta ; \beta)=\beta\left(1-\exp \left[-\left(g^{O}(\theta)-g_{\min }\right) / \beta\right]\right)$, and let $\bar{t}(\beta) \equiv \delta(\bar{\theta} ; \beta)$ for any given $\beta$; since $\delta(\bar{\theta} ; \beta)$ is increasing in $\beta$, so is $\bar{t}(\beta)$. Therefore the support of $H^{\delta}(t ; \beta)$ induced by $H(\theta)$ and $\delta(\theta ; \beta)$ is $[0, \bar{t}(\beta)]$. Then, fixing $\beta$ :

$$
H^{\delta}(t ; \beta) \equiv \operatorname{Pr}\left\{\delta\left(\theta_{i} ; \beta\right) \leq t\right\}=\operatorname{Pr}\left\{\theta \leq\left(g^{O}\right)^{-1}\left(\beta \ln \left(\beta /(\beta-t)+g_{\text {min }}\right)\right\}=H\left(\left(g^{O}\right)^{-1}\left(\beta \ln \left(\beta /(\beta-t)+g_{\text {min }}\right)\right) .\right.\right.
$$

Thus, $\partial H^{\delta}(t ; \beta) / \partial \beta=h(t)\left[\left(\left(g^{O}\right)^{-1}(t)\right)^{\prime}\left(\ln \left(\beta /(\beta-t)+g_{\min }\right)\right][\ln \beta+1-\ln (\beta-t)-\beta /(\beta-t)]\right.$, so that $\partial H^{\delta}(t ; \beta) / \partial \beta<0$ if and only if $\ln \beta+1-\ln (\beta-t)-\beta /(\beta-t)<0$. Note that $H^{\delta}(0 ; \beta)=0$ and $H^{\delta}(\bar{t}(\beta) ; \beta)$ $=\operatorname{Pr}\{\delta(\bar{\theta} ; \beta) \leq \bar{t}(\beta)\}=1$ for any given value of $\beta$, so we are interested in $\partial H^{\delta}(t ; \beta) / \partial \beta$ for $t \in(0, \bar{t}(\beta)) .{ }^{1}$

\footnotetext{
${ }^{1}$ Note that increasing $\beta$ increases the right end-point, so this means we must extend $H^{\delta}(t ; \beta)$ to be 1 on the interval $\left[\bar{t}(\beta), \bar{t}\left(\beta^{\prime}\right)\right]$ when we compare it to the distribution $H^{\delta}\left(t ; \beta^{\prime}\right)$, so that they are on the same support.
} 
Note that $\ln \beta+1-\ln (\beta-t)-\beta /(\beta-t)<0$ if and only if $\ln (\beta /(\beta-t))<1-\beta /(\beta-t)$ for $t$ in this open interval. Note that $t<\beta$ since $\left(1-\exp \left[-\left(g^{O}(\theta)-g_{\text {min }}\right) / \beta\right]\right)<1$. Thus, we may restate the problem as: is $\ln x<x-1$ for $x \geq 1$ ? In fact, the line $x-1$ is tangent to $\ln x$ at $x=1$, so $\ln x<x-1$ for $x>1$ and the two functions are equal at $x=1$. Therefore, $\partial H^{\delta}(t ; \beta) / \partial \beta<0$ for $t \in(0, \bar{t}(\beta))$, so that if $\beta^{\prime}$ $>\beta$, then $H^{\delta}\left(t ; \beta^{\prime}\right)<H^{\delta}(t ; \beta)$ for $t \in(0, \bar{t}(\beta))$; that is, $H^{\delta}\left(t ; \beta^{\prime}\right)$ FOSD $H^{\delta}(t ; \beta)$.

\section{Computational Results on the Effect of $\beta$ on $\alpha^{P O}$}

Table 1 below displays computational results for four density functions: 1) the Uniform density, with $h(\theta)=1 ; 2$ ) the Left Triangle density, with $h(\theta)=2-2 \theta$; 3) the Middle Triangle density, with $h(\theta)=4 \theta$ when $\theta \leq 1 / 2$, and $h(\theta)=4-4 \theta$ when $\theta>1 / 2$; and 4) the Right Triangle density, with $h(\theta)=2 \theta$. Notice that the Uniform density is a mean-preserving spread of the Middle Triangle density.

TABLE 1 - EFFECT OF $\beta$ ON $E\left(\delta^{2}\right) / E(\delta)$ FOR ALTERNATIVE DENSITIES OF $\theta$

\begin{tabular}{|c|c|c|c|}
\hline density $\downarrow$ & 0.5 & 1.0 & 2.0 \\
\hline Uniform & 0.40859 & 0.69264 & 1.14159 \\
\hline Left Triangle & 0.36996 & 0.61131 & 0.96546 \\
\hline Middle Triangle & 0.41363 & 0.69296 & 1.10361 \\
\hline Right Triangle & 0.43900 & 0.75341 & 1.22101 \\
\hline
\end{tabular}

Table 1 suggests that, for a given density, increasing $\beta$ increases $\alpha^{P O}$, so that $\Phi^{P O}\left(\alpha^{P O}\right)$ shifts up, associating more values of $\alpha$ with privacy than were associated with the lower value of $\beta$. Also, note that, holding $\beta$ constant, the computed values of $E\left(\delta^{2}\right) / E(\delta)$ increase as we move from the Left to the Middle to the Right Triangle distributions. Thus, Table 1 is consistent with the conjecture that a shift in $H$ to a new distribution $H^{\prime}$, where $H^{\prime}$ first-order stochastic dominates $H$, results in higher 
values of $\alpha^{P O}$ as well (i.e., upward shifts of $\Phi^{P O}$, too).

\section{Material on Interim Preferences over Policies $P$ and $O$}

This material pertains to Proposition 4. Two results follow from equation (5). First, comparing with equation (3), we see that $E\left(\Gamma^{P O}(\theta, \alpha)\right)=\Phi^{P O}(\alpha)$, so that when evaluated at $\alpha=\alpha^{P O}$, $E\left(\Gamma^{P O}\left(\theta, \alpha^{P O}\right), \alpha^{P O}\right)=0$. Since differentiating $\Gamma^{P O}\left(\theta_{i}, \alpha\right)$ shows that it is a monotonically decreasing function of $\theta_{i}$ for each value of $\alpha$, this implies that $\Gamma^{P O}\left(0, \alpha^{P O}\right)>0$ and $\Gamma^{P O}\left(\bar{\theta}, \alpha^{P O}\right)<0$, so that on an interim basis, if $\alpha=\alpha^{P O}$, then lower types will (interim) prefer $P$ to $O$ and higher types will (interim) prefer $O$ to $P$. Define two other values of $\alpha$, namely $\underline{\alpha}^{P O} \geq 0$ such that $\Gamma^{P O}\left(\bar{\theta}, \underline{\alpha}^{P O}\right)=0$ when $\mu \geq \bar{\theta}$ $(\delta(\bar{\theta} ; \beta))^{2} / 2 \beta$ (that is, the value of $\alpha$ such that all types will interim prefer $P$ to $O$ for any $\alpha \leq \underline{\alpha}^{P O}$; note that if $\mu<\bar{\theta}-(\delta(\bar{\theta} ; \beta))^{2} / 2 \beta$ then no such non-negative value exists $)$, and $\bar{\alpha}^{P O}$ such that $\Gamma^{P O}\left(0, \bar{\alpha}^{P O}\right)$ $=0$ (that is, the value of $\alpha$ such that all types will interim prefer $O$ to $P$ for any $\alpha \geq \bar{\alpha}^{P O}$ ). By construction, $\underline{\alpha}^{P O}<\alpha^{P O}<\bar{\alpha}^{P O}$. Furthermore, when $\alpha \leq \underline{\alpha}^{P O}$, the ex ante social preference for $P$ over $O$ is therefore reinforced by interim unanimity for $P$ over $O$, while when $\alpha \geq \bar{\alpha}^{P O}$, the ex ante social preference for $O$ over $P$ is reinforced by interim unanimity for $O$ over $P$. However, when $\alpha$ lies between $\underline{\alpha}^{P O}$ and $\bar{\alpha}^{P O}$, lower types prefer $P$ to $O$ while higher types prefer $O$ to $P$, so that for all $\alpha$ in the interval $\left(\underline{\alpha}^{P O}, \bar{\alpha}^{P O}\right)$ there is disagreement about the preferred policy at the interim stage, and there will not be unanimous reinforcement of any ex ante policy choice.

\section{E. Conflict Between Ex Ante and Interim Preferences}

To see the possibility of conflict between ex ante and interim preferences in a case wherein $O$ is ex ante preferred but $P$ is interim preferred by the median type, let $\theta^{P O}(\alpha)$ be the marginal type 
such that $\Gamma^{P O}\left(\theta^{P O}(\alpha), \alpha\right)=0$, for $\alpha \geq 0$. Note that $\theta^{P O}(\alpha)$ is decreasing in $\alpha$, and that $\theta^{P O}(0)>\mu$, the mean (and median) type if $H$ is symmetric. Thus, there is an $\alpha^{*}$ such that $\theta^{P O}\left(\alpha^{*}\right)=\mu$. It is straightforward to show that $\alpha^{*} \in\left(\alpha^{P O}, \bar{\alpha}^{P O}\right)$, so that for any value of $\alpha$ in the interval $\left(\alpha^{P O}, \alpha^{*}\right)$, the ex ante social payoff-maximizing choice of policy is $O$, but on an interim basis, the median type would prefer $P$ to $O$.

To see how the reverse conflict can occur, assume that $\alpha=0$. Since $\alpha^{P O}>0$, this means that society ex ante prefers $P$ to $O$. Since $\theta^{P O}(0)>\mu$, then any density $h$ whose median is to the right of $\theta^{P O}(0)$ implies that the median type prefers $O$ to $P$. Signaling type to gain esteem is sufficiently valuable to the median type (but is irrelevant in the case of the ex ante decision) for those types to interim prefer $O$ to $P$. This conflict between the ex ante and interim settings is summarized below. REMARK 2. Conflicting Ex Ante and Interim Preferences over Policies.

$h$ symmetric: There are values of $\alpha$ such that while a policy of publicity is ex ante socially preferred, the alternative policy of privacy is interim-preferred by the median type. $h$ sufficiently right-weighted: There are values of $\alpha$ such that while a policy of privacy is $e x$ ante socially preferred, a policy of publicity is interim-preferred by the median type.

\section{PROOF OF PROPOSITION 6(a):}

Proposition 6(a) provides the following ordering of the $\alpha$-values at which there is ex ante indifference between any two policies: $0<\alpha^{W O}<\alpha^{P O}<\alpha^{P W}$. To see that $0<\alpha^{W O}<\alpha^{P O}$, let:

$$
\eta(t) \equiv \int_{0}^{t}(\delta(\theta ; \beta))^{2} h(\theta) d \theta l \int_{0}^{t} \delta(\theta ; \beta) h(\theta) d \theta
$$

Then $\alpha^{W O}=\eta\left(\theta^{W}\right)$, which is clearly positive, while $\alpha^{P O}=\eta(\bar{\theta})$. It is straightforward to show that sgn $\left\{\eta^{\prime}(t)\right\}=\operatorname{sgn}\left\{\delta(t ; \beta) \int_{0}^{t} \delta(\theta ; \beta) h(\theta) d \theta-\int_{0}^{t}(\delta(\theta ; \beta))^{2} h(\theta) d \theta\right\}>0$ for all $t>0$. Therefore, it follows that $\alpha^{P O}=\eta(\bar{\theta})>\eta\left(\theta^{W}\right)=\alpha^{W O}$. 
To see that $\alpha^{P O}<\alpha^{P W}$, let

$$
v(s) \equiv \int_{s}^{\bar{\theta}}(\delta(\theta ; \beta))^{2} h(\theta) d \theta / \int_{s}^{\bar{\theta}} \delta(\theta ; \beta) h(\theta) d \theta .
$$

Then $\alpha^{P O}=v(0)$, while $\alpha^{P W}=v\left(\theta^{W}\right)$. It is straightforward to show that $\operatorname{sgn}\left\{v^{\prime}(s)\right\}=\operatorname{sgn}$ $\left\{\int_{s}^{\bar{\theta}}(\delta(\theta ; \beta))^{2} h(\theta) d \theta-\delta(s ; \beta) \int_{s}^{\bar{\theta}} \delta(\theta ; \beta) h(\theta) d \theta\right\}>0$ for all $s<\bar{\theta}$. Therefore, it follows that $\alpha^{P O}=v(0)$ $<v\left(\theta^{W}\right)=\alpha^{P W}$

\section{F. Material on Interim Preferences over Policies $P, O$ and $W$}

Throughout this discussion we assume that $\theta^{W} \in(0, \bar{\theta})$; if not, then the policy $W$ coincides with either $O$ or $P$ and there are not three distinct policies to be compared.

Recall that the conditional mean is $\mu\left(\theta^{W}\right)=\int_{\mathcal{J}} t h(t) d t / H\left(\theta^{W}\right)$, where $\mathcal{T}=\left[0, \theta^{W}\right]$. Furthermore, let $E\left(g^{O}-g^{P}\right)$ denote the expected distortion under a policy of $O$ versus a policy of $P$, and similarly for $E\left(g^{W}-g^{P}\right)$ and $E\left(g^{O}-g^{W}\right)$. Then:

(a) $E\left(g^{O}-g^{P}\right)=\int \delta(t ; \beta) h(t) d t$, where the integral is taken over $[0, \bar{\theta}]$;

(b) $E\left(g^{W}-g^{P}\right)=\int_{\mathscr{T} \mathcal{C}} \delta(t ; \beta) h(t) d t$, where the integral is taken over $\mathcal{T} \mathcal{C}=\left[\theta^{W}, \bar{\theta}\right]$;

(c) $E\left(g^{O}-g^{W}\right)=\int_{\mathcal{T}} \delta(t ; \beta) h(t) d t$, where the integral is taken over $\mathcal{T}=\left[0, \theta^{W}\right]$.

The integral in part (a) reflects the fact that every type (except the lowest) distorts her action under a policy of $O$ while no type distorts her action under a policy of $P$. The integral in part (b) reflects the fact that only those types in $\mathcal{T C}=\left[\theta^{W}, \bar{\theta}\right]$ distort their actions. Finally, the integral in part (c) reflects the fact that only those types in $\mathcal{T}=\left[0, \theta^{W}\right]$ do not distort their actions.

These definitions allow us to summarize the type-specific value of one policy over another. Let $\Gamma^{P O}\left(\theta_{i}, \alpha\right) \equiv V_{i}\left(g^{P}\left(\theta_{i}\right), \theta_{i}, \mu, G^{P}\right)-V_{i}\left(g^{O}\left(\theta_{i}\right), \theta_{i}, \theta_{i}, G^{O}\right)$ denote the type-specific value of a policy of privacy over a policy of publicity. Then: 


$$
\Gamma^{P O}\left(\theta_{i}, \alpha\right)=\beta\left(\mu-\theta_{i}\right)+\left(\delta\left(\theta_{i} ; \beta\right)\right)^{2} / 2-\alpha M E\left(g^{O}-g^{P}\right)
$$

Similarly, let $\Gamma^{P W}\left(\theta_{i}, \alpha\right) \equiv V_{i}\left(g^{P}\left(\theta_{i}\right), \theta_{i}, \mu, G^{P}\right)-V_{i}\left(g^{W}\left(\theta_{i}\right), \theta_{i}, \widetilde{\theta}_{i}, G^{W}\right)$ denote the type-specific value of a policy of privacy over a policy of waiver. Then:

$$
\begin{aligned}
\Gamma^{P W}\left(\theta_{i}, \alpha\right) & =\beta\left(\mu-\mu\left(\theta^{W}\right)\right)-\alpha M E\left(g^{W}-g^{P}\right) \text { for } \theta_{i}<\theta^{W} ; \text { and } \\
& =\beta\left(\mu-\theta_{i}\right)+\left(\delta\left(\theta_{i} ; \beta\right)\right)^{2} / 2-\alpha M E\left(g^{W}-g^{P}\right), \text { for } \theta_{i} \geq \theta^{W} .
\end{aligned}
$$

Finally, let $\Gamma^{W O}\left(\theta_{i}, \alpha\right) \equiv V_{i}\left(g^{W}\left(\theta_{i}\right), \theta_{i}, \widetilde{\theta}_{i}, G^{W}\right)-V_{i}\left(g^{O}\left(\theta_{i}\right), \theta_{i}, \theta_{i}, G^{O}\right)$ denote the type-specific value of a policy of waiver over a policy of publicity. Then:

$$
\begin{aligned}
\Gamma^{W O}\left(\theta_{i}, \alpha\right) & =\beta\left(\mu\left(\theta^{W}\right)-\theta_{i}\right)+\left(\delta\left(\theta_{i} ; \beta\right)\right)^{2} / 2-\alpha M E\left(g^{O}-g^{W}\right), \text { for } \theta_{i}<\theta^{W} ; \text { and } \\
& =-\alpha M E\left(g^{O}-g^{W}\right), \text { for } \theta_{i} \geq \theta^{W}, \text { for } \theta_{i} \geq \theta^{W} .
\end{aligned}
$$

The functions $\Gamma^{P O}\left(\theta_{i}, \alpha\right), \Gamma^{P W}\left(\theta_{i}, \alpha\right)$, and $\Gamma^{W O}\left(\theta_{i}, \alpha\right)$ are continuous in both arguments and strictly decreasing in $\alpha$; the latter two functions have portions that are constant with respect to $\theta_{i}$, but they are strictly decreasing in $\theta_{i}$ over the non-constant regions.

We first determine conditions under which there will be non-trivial sets of types who prefer each policy in a binary comparison. In particular, let $\bar{\alpha}^{I J}$, for $I J=P O, P W, W O$, be the value of $\alpha$ for which $\theta_{i}=0$ is indifferent between policy $I$ and policy $J$ (for this and any higher value of $\alpha$, policy $J$ will be preferred to policy $I$ for all types). Then $\bar{\alpha}^{I J}$ is defined uniquely by $\Gamma^{I J}\left(0, \bar{\alpha}^{I J}\right)=0$, yielding:

$$
\begin{aligned}
& \bar{\alpha}^{P O}=\beta \mu /\left(M E\left(g^{O}-g^{P}\right)\right) ; \\
& \bar{\alpha}^{P W}=\beta\left(\mu-\mu\left(\theta^{W}\right)\right) /\left(M E\left(g^{W}-g^{P}\right)\right) ; \\
& \bar{\alpha}^{W O}=\beta \mu\left(\theta^{W}\right) /\left(M E\left(g^{O}-g^{W}\right)\right) .
\end{aligned}
$$

Provided that $\alpha<\min \left\{\bar{\alpha}^{I J}\right\}$, there will be at least some (low) types who prefer policy $I$ to policy $J$ in a binary comparison. In order to have at least some (high) types who prefer policy $J$ to policy $I$ in a binary comparison, it must be that $\Gamma^{I J}(\bar{\theta}, \alpha)<0$; our hypothesis that $\theta^{W}<\bar{\theta}$ is enough to 
guarantee that this holds for all $\alpha>0$.

CLAIM 1: If $0<\alpha<\min \left\{\bar{\alpha}^{I J}\right\}$, then:

(i) there exists a unique $\theta^{I J}(\alpha) \in(0, \bar{\theta})$ such that $\Gamma^{I J}\left(\theta^{I J}(\alpha), \alpha\right)=0$;

(ii) moreover, $\theta^{W O}(\alpha)<\theta^{W}<\theta^{P W}(\alpha)$ and $\theta^{W O}(\alpha)<\theta^{P O}(\alpha)<\theta^{P W}(\alpha)$.

\section{PROOF OF CLAIM 1:}

By construction, if $0<\alpha<\min \left\{\bar{\alpha}^{I J}\right\}$, then $\Gamma^{I J}(0, \alpha)>0$ and $\Gamma^{I J}(\bar{\theta}, \alpha)<0$, for all $I J$. First consider $I J=P O$. The function $\Gamma^{P O}(\theta, \alpha)$ is continuous and strictly decreasing in $\theta$; therefore there exists a unique value $\theta^{I J}(\alpha) \in(0, \bar{\theta})$ such that $\Gamma^{I J}\left(\theta^{I J}(\alpha), \alpha\right)=0$. Next consider $I J=P W$. The function $\Gamma^{P W}(\theta$, $\alpha)$ is constant at a positive level for $\theta_{i}<\theta^{W}$, and $\Gamma^{P W}(\theta, \alpha)=\Gamma^{P O}(\theta, \alpha)+E\left(g^{O}-g^{W}\right)$ for $\theta_{i} \geq \theta^{W}$. Since this is a continuous and strictly decreasing function, there is a unique value $\theta^{P W}(\alpha) \in\left(\theta^{W}, \bar{\theta}\right)$ such that $\Gamma^{P W}\left(\theta^{P W}(\alpha), \alpha\right)=0$. Moreover, this implies that $\Gamma^{P O}\left(\theta^{P W}(\alpha), \alpha\right)=-E\left(g^{O}-g^{W}\right)<0$, so $\theta^{P O}(\alpha)<\theta^{P W}(\alpha)$. Finally, consider $I J=W O$. The function $\Gamma^{W O}(\theta, \alpha)$ is constant at a negative level for $\theta_{i} \geq \theta^{W}$; it is a continuous and strictly decreasing function for $\theta_{i}<\theta^{W}$. Therefore, there is a unique value $\theta^{W O}(\alpha) \in$ $\left(0, \theta^{W}\right)$ such that $\Gamma^{W O}\left(\theta^{W O}(\alpha), \alpha\right)=0$. Moreover, evaluating $\Gamma^{P O}$ at this level yields $\Gamma^{P O}\left(\theta^{W O}(\alpha), \alpha\right)=$ $\Gamma^{P W}(0, \alpha)>0$, so $\theta^{W O}(\alpha)<\theta^{P O}(\alpha)$.

Note that for the special case of $\alpha=0$ the claim above still holds with the following minor modifications. Now the function $\Gamma^{W O}(\theta, \alpha)$ starts out positive and declines to zero at $\theta^{W}$; moreover, it remains constant at zero for $\theta_{i} \geq \theta^{W}$. Thus, the equation $\Gamma^{W O}\left(\theta^{W O}(\alpha), \alpha\right)=0$ is satisfied by all members of the set $\left[\theta^{W}, \bar{\theta}\right]$; we take the left-most element as $\theta^{W O}(\alpha)$, and thus $\theta^{W O}(\alpha)=\theta^{W}$. The rest of the claim continues to hold as stated. 
Given the ordering $\theta^{W O}(\alpha)<\theta^{P O}(\alpha)<\theta^{P W}(\alpha)$ derived above, it is straightforward to show that no type finds $W$ to be the best policy. The preference orderings are as follows and are illustrated in Figure 3 in the main text:

For $\theta \in\left[0, \theta^{W O}(\alpha)\right) \quad P \succ W \succ O \quad$ (with $W \sim O$ at $\theta^{W O}(\alpha)$ )

For $\theta \in\left(\theta^{W O}(\alpha), \theta^{P O}(\alpha)\right) \quad P \succ O \succ W \quad$ (with $P \sim O \succ W$ at $\theta^{P O}(\alpha)$ )

For $\theta \in\left(\theta^{P O}(\alpha), \theta^{P W}(\alpha)\right) \quad O \succ P \succ W \quad\left(O \succ P \sim W\right.$ at $\left.\theta^{P W}(\alpha)\right)$

For $\theta \in\left(\theta^{P W}(\alpha), \bar{\theta}\right] \quad O \succ W \succ P$

Now we relax the assumption that $\alpha<\min \left\{\bar{\alpha}^{I J}\right\}, I J=P O, P W, W O$. It is straightforward to show that $\bar{\alpha}^{P O}$ must lie between $\bar{\alpha}^{P W}$ and $\bar{\alpha}^{W O}$, but we are unable to determine in general whether $\bar{\alpha}^{P W}$ $<\bar{\alpha}^{W O}$ or $\bar{\alpha}^{W O}<\bar{\alpha}^{P W}$ (however, if $\bar{\alpha}^{W O}<\bar{\alpha}^{P W}$, then $W$ can never be interim-optimal for any type because $\Gamma^{W O}(0, \alpha)<0$, implying that $O$ is preferred to $W$ for all types $)$.

As claimed in the text, there are conditions under which some types will most-prefer a policy of $W$; these conditions are now described. First, it can be shown that $\bar{\alpha}^{P W}<\bar{\alpha}^{W O}$ for the case in which $\theta$ is distributed uniformly on $[0, \bar{\theta}]$. For $\bar{\alpha}^{P W}<\alpha<\bar{\alpha}^{W O}$, all types strictly prefer $P$ to $W$, while those in $\left[0, \theta^{W O}(\alpha)\right)$ also strictly prefer $W$ to $O$. So it is possible for some types to interim-prefer $W$ to both $P$ and $O$ (however, this set is limited by the fact that $\theta^{W O}(\alpha)<\theta^{W}$ still holds). Notice that the types who interim-prefer $W$ to both $P$ and $O$ will exercise privacy under a policy of $W$ (since they are $<$ $\left.\theta^{W}\right)$, but hope to gain both from higher types who also choose privacy and from the disclosures and distortions of even higher types. 